Originalien

Med Klin Intensivmed Notfmed 2022 - 117: 144-151

https://doi.org/10.1007/s00063-021-00820-5

Eingegangen: 6. Januar 2021

Angenommen: 7. März 2021

Online publiziert: 20. April 2021

(c) Der/die Autor(en) 2021

Redaktion

M. Buerke, Siegen

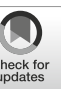

\section{Zusatzmaterial online}

Zusätzliche Informationen sind in der Onlineversion dieses Artikels (https://doi.org/ 10.1007/s00063-021-00820-5) verfügbar. Sie enthält den Studienfragebogen. Beitrag und Zusatzmaterial stehen Ihnen auf www. springermedizin.de zur Verfügung. Bitte geben Sie dort den Beitragstitel in die Suche ein, das Zusatzmaterial finden Sie beim Beitrag unter „Ergänzende Inhalte“.

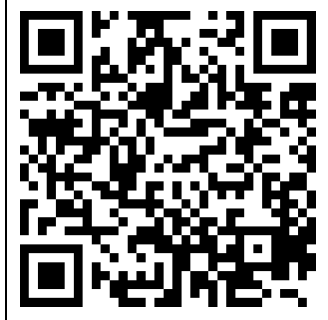

\section{Hintergrund und Fragestellung}

In der Versorgung von medizinischen Akutfällen wird die sektorale Trennung des deutschen Gesundheitssystems besonders deutlich. Derzeit existieren 3 Systeme parallel: Rettungsdienst, ärztlicher Bereitschaftsdienst (ÄBD) der Kassenärztlichen Vereinigung und Notaufnahmen an Krankenhäusern [1,14,27]. Diese Systeme sind als gegenseitige Ergänzung konzipiert, werden allerdings unabhängig voneinander organisiert $[8,9,14,27]$. Die Zuordnung erfolgt momentan durch den Patienten eigenständig und nicht an-

Bibiana Metelmann' ${ }^{1}$ - Peter Brinkrolf' $\cdot$ Marian Kliche ${ }^{1} \cdot$ Marcus Vollmer $^{2}$. Klaus Hahnenkamp' · Camilla Metelmann'

'Klinik für Anästhesiologie, Anästhesie, Intensiv-, Notfall- und Schmerzmedizin, Universitätsmedizin Greifswald, Greifswald, Deutschland

${ }^{2}$ Institut für Bioinformatik, Universitätsmedizin Greifswald, Greifswald, Deutschland

\title{
Rettungsdienst, kassenärztlicher Notdienst oder Notaufnahme
}

\author{
Es gelingt der Bevölkerung nur \\ unzureichend, die richtige Ressource für \\ medizinische Akutfälle zu wählen
}

hand einer Einschätzung durch medizinisches Personal [1, 4].

Die Bevölkerung muss ausreichend über diese 3 Systeme informiert sein, um bei der Auswahl der notwendigen Ressource nicht nur subjektiven Präferenzen oder emotionalen Aspekten zu folgen [5, $10,25,30]$. Nur so können die Patienten bei medizinischen Problemen kenntnisbasiert das für diesen Fall adäquate System wählen und kontaktieren.

Hieraus ergab sich die Frage, ob das Wissen über diese Systeme in der Bevölkerung ausreichend hoch vorhanden ist und ob die Bevölkerung die Dringlichkeit verschiedener Situationen adäquat einschätzen kann.

\section{Studiendesign und Unter- suchungsmethoden}

Im Rahmen dieser Querschnittsstudie wurde entsprechend den Richtlinien der GESIS, Leibniz-Institut für Sozialwissenschaften, eine telefonische Befragung einer Stichprobe der deutschen Bevölkerung durchgeführt [12]. Hierzu wurden vom 11. Juni 2018 bis zum 26. August 2018 von Montag bis Freitag von 9.00 bis 21.00 Uhr und Samstag von 10.00 bis 20.00 Uhr mit einer DualFrame-Stichprobe in gleichem Anteil Festnetz- und Mobilfunknummern, die nach dem Gabler-Häder-Design generiert waren, über „random digit dialing“ angerufen [11]. Dabei wurden bis zu
8 Kontaktversuche zum Erreichen eines Anschlusses unternommen und Telefonnummern in 100er-Blöcken genutzt [11]. Ausgeschlossen wurden nichtvergebene und geschäftliche Rufnummern. Eingeschlossen in die Befragung wurden Personen, die der Befragung zustimmten, nach eigenen Angaben mindestens 16 Jahre alt waren, fließend Deutsch sprachen und ihren Hauptwohnsitz in Deutschland hatten. Zur standardisierten Befragung wurden alle Telefonate anhand eines Fragebogens durch nur einen Untersucher durchgeführt.

Um die Kenntnisse und das Vorgehen bei medizinischen Akutsituationen praxisnah $\mathrm{zu}$ prüfen, wurden während des Telefonats in randomisierter Reihenfolge 6 fiktive Szenarien mit medizinischen Akutsituationen als Tonbandaufnahme eingespielt. Dabei waren jeweils 2 Szenarien so ausgelegt, dass eine Alarmierung des Rettungsdiensts bzw. des kassenärztlichen Notdiensts bzw. ein Abwarten und ggf. eine Vorstellung beim Hausarzt im Verlauf indiziert waren. Die Szenarien wurden durch die Autoren entwickelt und in einem Pretest durch Notärzte, Rettungsdienstmitarbeiter, Leitstellendisponenten und Sprachwissenschaftler validiert. Folgende Szenarien wurden konstruiert: eine Apoplexsymptomatik mit Hemiparese, ein typisches Beschwerdebild eines Myokardinfarkts, eine Lumboischalgiesymptomatik, ein persistierender Harn- 


\begin{tabular}{|l|l|l}
\hline Tab. 1 Stichprobenausschöpfung & Absolut & Relativ (in \%) \\
\hline 1. Insgesamt gewählte Nummern & 14.000 & $100 \%=14.000$ \\
\hline Nummer nicht vergeben & 8043 & 57,5 \\
\hline Geschäftliche Rufnummer & 265 & 1,9 \\
\hline Fax & 185 & 1,3 \\
\hline Summe der im ersten Schritt ausgeschlossenen Nummern & 8493 & 60,7 \\
\hline 2. Existente und private Nummern & 5507 & $100 \%=5507$ \\
\hline Stets Anrufbeantworter & 1776 & 32,2 \\
\hline Nie abgehoben & 1517 & 27,5 \\
\hline Einschlusskriterien nicht erfüllt & 93 & 1,7 \\
\hline Vereinbarter Termin nicht eingehalten & 322 & 5,8 \\
\hline Aufgelegt vor Erfragung des Einverständnisses & 270 & 4,9 \\
\hline Summe der im zweiten Schritt ausgeschlossenen Nummern & 3978 & 72,2 \\
\hline 3. Infrage kommende Nummern & 1529 & $100 \%=1529$ \\
\hline Nonresponse - Befragung abgelehnt & 806 & 52,7 \\
\hline Nonresponse - Befragung abgebrochen & 15 & 1,0 \\
\hline Realisierte Interviews & 708 & 46,3 \\
\hline Von 14.000 Nummern waren 1529 infrage kommende Nummern vorhanden und 708 (46,3\%) Befra- \\
\hline gungen wurden vollständig durchgeführt & & \\
\hline
\end{tabular}

wegsinfekt mit Schmerzen, ein zufällig gemessener Bluthochdruck ohne Symptome und ein seit 2 Tagen bestehender grippaler Infekt. Die Befragten wurden gebeten, sich in die Rolle eines Patienten zu versetzen, der an einem Samstag diese Situation erlebt. Nach jedem einzelnen Szenario wurden die Teilnehmer zur subjektiven Einschätzung der Situation und zum weiteren Vorgehen befragt. Hierfür wurde erfasst, ob sie kurzfristig (innerhalb der nächsten Minuten bis Stunden) medizinische Hilfe bräuchten und, falls $\mathrm{ja}$, an wen sie sich wenden würden. Außerdem wurde gefragt, für wie akut $(1=$ nicht akut bis $10=$ sehr akut) sie die Situation einschätzten, und dies anschließend in die Kategorien $1=$ nicht dringlich, $2-4=$ leicht dringlich, 5-7= mittel dringlich, $8-10=\mathrm{sehr}$ dringlich umcodiert. Zusätzlich wurde die Bekanntheit der unterschiedlichen Ressourcen bei medizinischen Akutfällen erfasst. Der vollständige Fragebogen inklusive Beschreibung der Szenarien befindet sich im elektronischen Zusatzmaterial online.

Die Fallzahlplanung ergab, aufbauend auf einer Studie von Kirkby, bei einer konservativen Schätzung einer korrekten Beurteilungsrate der Fallszenarien von $50 \%$ eine erforderliche Mindestanzahl von 706 Interviews, um die Spannweite des $95 \%$-Konfidenzintervalls auf maximal 7,5\% zu begrenzen [13]. Die statistische Analyse erfolgte mit der Software RStudio $^{\circledast}$ (RStudio PBC, Boston, U.S.A.). Ein positives Votum der Ethikkommission der Universitätsmedizin Greifswald (Interne Reg. Nr.: BB 078/18) liegt vor.

\section{Ergebnisse}

Im Rahmen der Befragung wurden 14.000 Telefonnummern angewählt. Es konnten 708 vollständige Interviews realisiert werden. - Tab. 1 zeigt die Stichprobenausschöpfung nach Anwendung der Ein- und Ausschlusskriterien.

Durch das Gabler-Häder-Design wurde eine repräsentative Stichprobe der deutschen Bevölkerung angestrebt. In der Stichprobe waren Personen aus allen Bundesländern vertreten. Bei der Altersverteilung gab es keinen signifikanten Unterschied zwischen der Telefonstichprobe und der Bevölkerungsstatistik des Statistischen Bundesamts ( $p=0,099$; - Abb. 1). Jedoch sind in der Stichprobe mit 57,6\% $(n=408)$ signifikant mehr Frauen als Männer $(42,4 \% ; n=300)$ vertreten im Vergleich zur deutschen Bevölkerung mit 50,7\% ( $n=36,54$ Mio.; $p<0,05)$. Zusätzlich lebten signifikant mehr Personen der Stichprobe in ländlichen Regionen (unter 4999 Einwohner) mit $26 \%(n=184)$ im Vergleich zu $14 \%$ $(n=11,74$ Mio.; $p<0,05)$. Der prozentuale Anteil an Personen mit hohem Bildungsabschluss war mit $29 \%$ signifikant höher als in der Bevölkerung mit $13 \%(p<0,05)$.

Bei den Szenarien Apoplex und Myokardinfarkt hielten 98,0\% $(n=694)$ bzw. 94,5\% $(n=669)$ der Befragten medizinische Hilfe für nötig. Bei einem Harnwegsinfekt gaben 58,6\% der Befragten an, dass sie kurzfristig medizinische Hilfe benötigen (siehe 0 Abb. 2).

Schätzten die Befragten eine medizinische Hilfe als erforderlich ein, wurden sie gefragt, welche Ressource sie wählen würden. • Abb. 3 zeigt, dass bei sehr dringlichen Szenarien 74,5\% $(n=1015)$ den Rettungsdienst alarmieren, jedoch 6,7\% $(n=91)$ den $\mathrm{ÄBD}$ alarmieren würden und sich $18,9 \%$ $(n=257)$ selbstständig in der Notaufnahme vorstellen würden. Bei Szenarien mit mittlerer Dringlichkeit würden $38,2 \%(n=245)$ den $\ddot{B} B D$ alarmieren, aber $45,9 \%(n=294)$ sich selbstständig in der Notaufnahme vorstellen. Ähnliche Prozentwerte zeigen sich bei Fällen mit leichter Dringlichkeit $(39,7 \%, n=52$ ÄBD und $46,6 \%, n=61$ Notaufnahme).

- Abb. 4 illustriert die von den Teilnehmern subjektiv eingeschätzte Dringlichkeit in Relation zur tatsächlichen Dringlichkeit. Die korrekte Einschätzung der Dringlichkeit gelang beim Szenario Apoplex 83,8\% $(n=593)$ der Befragten, beim Myokardinfarkt $77 \%$ $(n=545)$, beim Rückenschmerz $45,1 \%$ $(n=319)$, beim Harnwegsinfekt $56,8 \%$ $(n=402)$, beim zufällig entdeckten Hypertonus $66,1 \%(n=468)$ und bei dem grippalen Infekt $79,9 \%(n=565)$ der Befragten.

Als Ressourcen, unter welcher man medizinische Hilfe bekommen könnte, nannten 94,5\% $(n=669)$ der Befragten den Rettungsdienst/Notruf eigenständig und 99,7\% $(n=706)$ kannten diesen auf Nachfrage. 87,9\% $(n=622)$ der Befragten konnten die 112 als gültige Telefonnummer nennen (siehe @ Abb. 5). Den ÄBD nannten $53,7 \%(n=380)$ der Befragten eigenständig und $75,3 \%(n=533)$ kannten diesen auf Nachfrage. Eine gültige Telefonnummer (116117 oder regionale $\mathrm{Te}$ - 
Med Klin Intensivmed Notfmed 2022 • 117:144-151 https://doi.org/10.1007/s00063-021-00820-5

(c) Der/die Autor(en) 2021

\section{B. Metelmann · P. Brinkrolf · M. Kliche · M. Vollmer · K. Hahnenkamp $\cdot$ C. Metelmann}

\section{Rettungsdienst, kassenärztlicher Notdienst oder Notaufnahme. Es gelingt der Bevölkerung nur unzureichend, die richtige Ressource für medizinische Akutfälle zu wählen}

\section{Zusammenfassung}

Hintergrund. Bei medizinischen Akutfällen entscheiden Patienten eigenständig, ob sie den Rettungsdienst bzw. den ärztlichen Bereitschaftsdienst der kassenärztlichen Vereinigung anrufen oder sich in der Notaufnahme vorstellen.

Fragestellung. Gelingt der Bevölkerung die angemessene Zuordnung verschiedener dringlicher Erkrankungen zu den unterschiedlichen Systemen? Material und Methoden. In einer deutschlandweiten, anonymen telefonischen Befragung nach dem Gabler-Häder-Design im Sommer 2018 wurden 708 Personen jeweils 6 verschiedene Szenarien mit medizinischen Akutfällen geschildert. Die Befragten wurden gebeten anzugeben, ob sie kurzfristige medizinische Hilfe für erforderlich hielten. Zusätzlich wurde die subjektive Dringlichkeit der einzelnen Szenarien sowie die Kenntnis der Telefonnummern von Rettungsdienst und ärztlichem Bereitschaftsdienst erhoben. Ergebnisse. Die Dringlichkeit der Szenarien wurde häufig fehleingeschätzt: bei Szenarien hoher Dringlichkeit zu 20\%, bei mittlerer Dringlichkeit zu $50 \%$ und bei leichter Dringlichkeit zu $27 \%$. Zusätzlich misslang einigen Befragten die Ressourcenwahl, wenn sie medizinische Hilfe für erforderlich hielten: $25 \%$ würden bei einem Apoplex bzw. Myokardinfarkt keinen Rettungsdienst rufen. Bei Erkrankungen mittlerer Dringlichkeit würden mehr Befragte eigenständig in die
Notaufnahme gehen (38\%), als den ärztlichen Bereitschaftsdienst zu alarmieren (46\%). Diskussion. Das Wissen der Bevölkerung über die verschiedenen Ressourcen bei medizinischen Akutfällen und die Fähigkeit, die Dringlichkeit adäquat einzuschätzen, scheint nicht ausreichend zu sein. Die Lösung könnte neben einer Steigerung der Gesundheitskompetenz eine gemeinsame Telefonnummer für Rettungsdienst und ärztlichen Bereitschaftsdienst mit einheitlichem Abfragetool und Ressourcenzuordnung sein.

Schlüsselwörter

Notarzt · Ärztlicher Bereitschaftsdienst . Notfall - Gemeinsames Notrufleitsystem . Telefonumfrage

\section{Emergency medical service, medical on-call service, or emergency department. Germans unsure whom to contact in acute medical events}

\section{Abstract}

Background. In medical events, patients have to independently decide whom to contact: emergency medical services, medical on-call service or emergency department.

Objectives. Are Germans able to assess the urgency of medical events and choose the correct resource?

Materials and methods. In 2018 a nationwide anonymous telephone survey was done in Gabler-Haeder design. In all, 708 interviewees were presented with six medical scenarios. Participants were asked to rate urgency and to assess whether medical help was necessary within minutes to hours. Telephone numbers of emergency medical services and medical on-call service were inquired.

Results. Urgency of different scenarios was often misjudged: in cases with high, medium, and low urgency the misjudgement rate were 20,50 , and $27 \%$, respectively. If medical help was rated as necessary, some participants chose the wrong service: $25 \%$ would not call an ambulance in stroke or myocardial infarction. In cases with medium urgency, more respondents chose to consult an emergency department (38\%) than to call medical on-call service (46\%).
Conclusions. Knowledge regarding different options for treatment of medical events and competence to assess urgency seem to be too low. Beside efforts to increase health literacy, one solution might be to introduce a joint telephone number for emergency medical services and medical on-call service with a uniform assessment tool and appropriate allocation.

Keywords

Prehospital emergency medicine - First aid . Emergency - Emergency number · Telephone survey lefonnummer) nannten 17,9\% $(n=127)$ der Teilnehmer.

\section{Diskussion}

Im deutschen Gesundheitssystem kann ein Patient bei neu aufgetretenen Beschwerden eigenständig entscheiden, welche Gesundheitseinrichtung er kontaktiert: den Rettungsdienst, den ärztlichen Bereitschaftsdienst oder die Notaufnahme [4]. Zusätzlich kann er auch zunächst abwarten und keine medizinische Hilfe anfordern. Um diese Ent- scheidung eigenständig sicher treffen zu können, muss der Patient ausreichend über diese Ressourcen informiert sein und zusätzlich die Erkrankungsschwere adäquat einschätzen können [25].

Diese Telefonumfrage zeigt, dass $12 \%$ der Befragten die Nummer 112 zur Alarmierung des Rettungsdienstes nicht kannten. Diese Zahl scheint über die letzten 15 Jahre konstant zu bleiben [19].

Der Bevölkerung scheint es schwer zu fallen, die Dringlichkeit der Situation adäquat einzuschätzen. In der Telefon- befragung haben bei der Schilderung typischer Beschwerden eines Apoplex und eines Myokardinfarkts $20 \%$ die Dringlichkeit unterschätzt. Dies deckt sich mit den Ergebnissen einer Telefonbefragung von 2013 [16]. Die Fehleinschätzung der Dringlichkeit kann zu erheblichen Zeitverzögerungen bis zum ersten medizinischen Kontakt und der erforderlichen Therapie führen. In unserer Befragung riefen $25 \%$ der Befragten bei diesen lebensbedrohlichen Szenarien nicht den Rettungsdienst. 

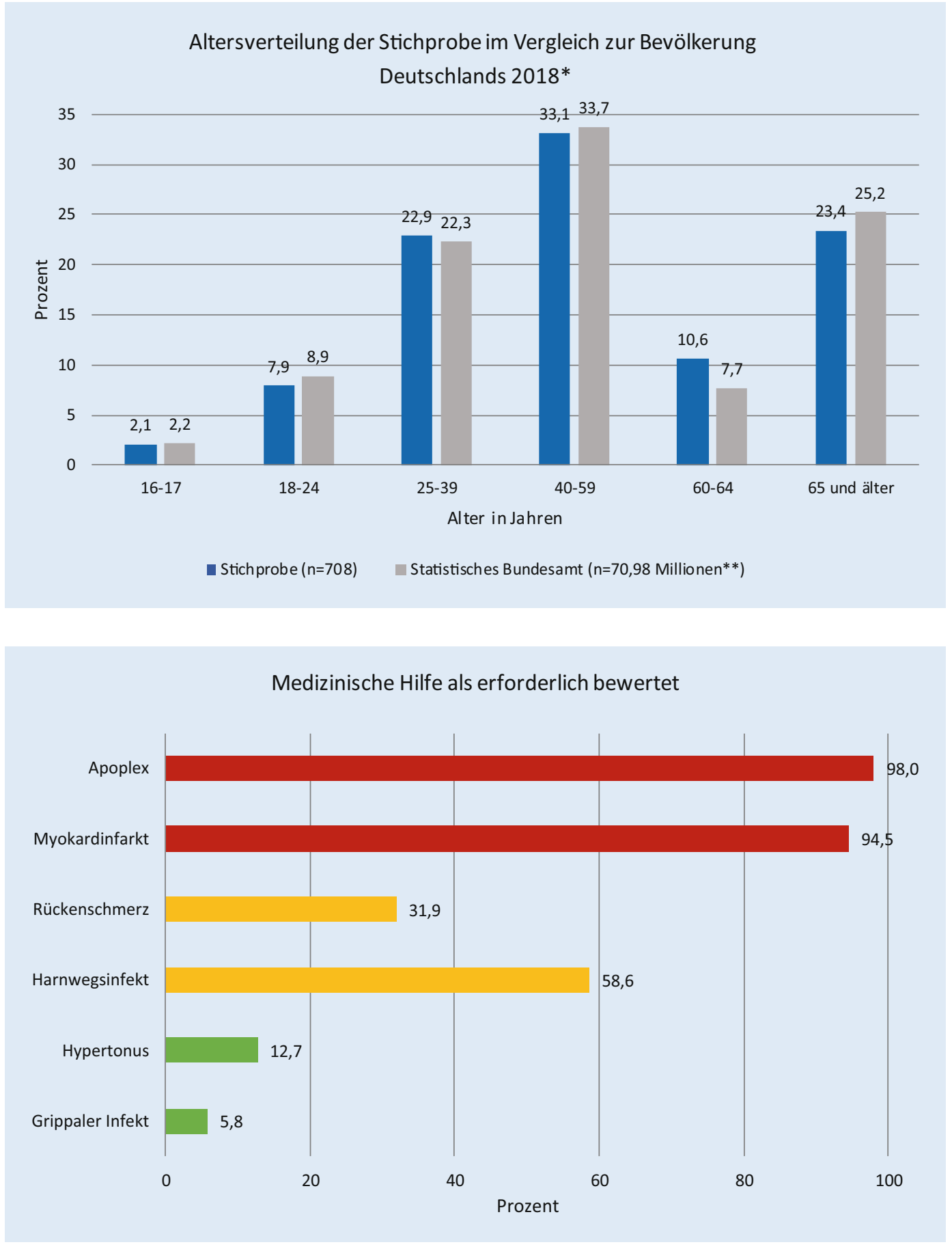

Abb. $1 \triangleleft$ Altersverteilung der Stichprobe im Vergleich zur Bevölkerung Deutschlands. ${ }^{*}$ Daten des Statistischen Bundesamts (Stand 31.12.2018); **2018 lebten insgesamt 83,02 Mio. Personen in Deutschland; das 1.-16. Lebensjahr wurde von der Gesamtbevölkerung subtrahiert (d.h. 12,04 Mio.) und die Altersverteilungen prozentual angepasst
Abb. $2 \triangleleft$ Hinzuziehen kurzfristiger medizinischer Hilfe aufgeschlüsselt nach Szenarien. Anteil der Befragten bei den verschiedenen Szenarien, die eine kurzfristige medizinische Hilfe für erforderlich hielten
Gleichzeitig wird die Dringlichkeit bei nicht-/leicht dringlichen Fälle in circa $30 \%$ überschätzt und bei mitteldringlichen Fällen gelang weniger als der Hälfte der Befragten die richtige Dringlichkeitseinstufung. Ähnliche Ergebnisse zeigen auch Studien zu Notaufnahmepatienten $[10,24,30,31]$. So schätzten sich $90 \%$ der Patienten, die sich fußläufig in der Notaufnahme vorstellen, als Notfall ein, wobei in 48-68\% der Fälle die Dringlichkeit der Behandlung durch die medizinischen Laien im Vergleich zu Notaufnahmeärzten höher eingeschätzt wurde [20, 30, 31]. Erfahrungen aus der Schweiz zeigen, dass $70 \%$ der Anrufer bei der medizinischen computerassistierten Telefontriage die Dringlichkeit ihrer Beschwerden falsch bewerteten [17]. Und nur bei $2 \%$ der Anrufer, die eine Notaufnahme aufsuchen wollten, war dies tatsächlich indiziert [17].

Obwohl eine Alarmierung des ÄBD indiziert gewesen wäre, würden bei mitteldringlichen Fällen mit 45,9\% mehr Personen eigenständig die Notaufnahme aufsuchen als den ÄBD (38,2\%) zu alarmieren. Und auch bei den leicht bzw. nichtdringlichen Fällen gehen mehr Personen in die Notaufnahme $(46,6 \%)$, als 


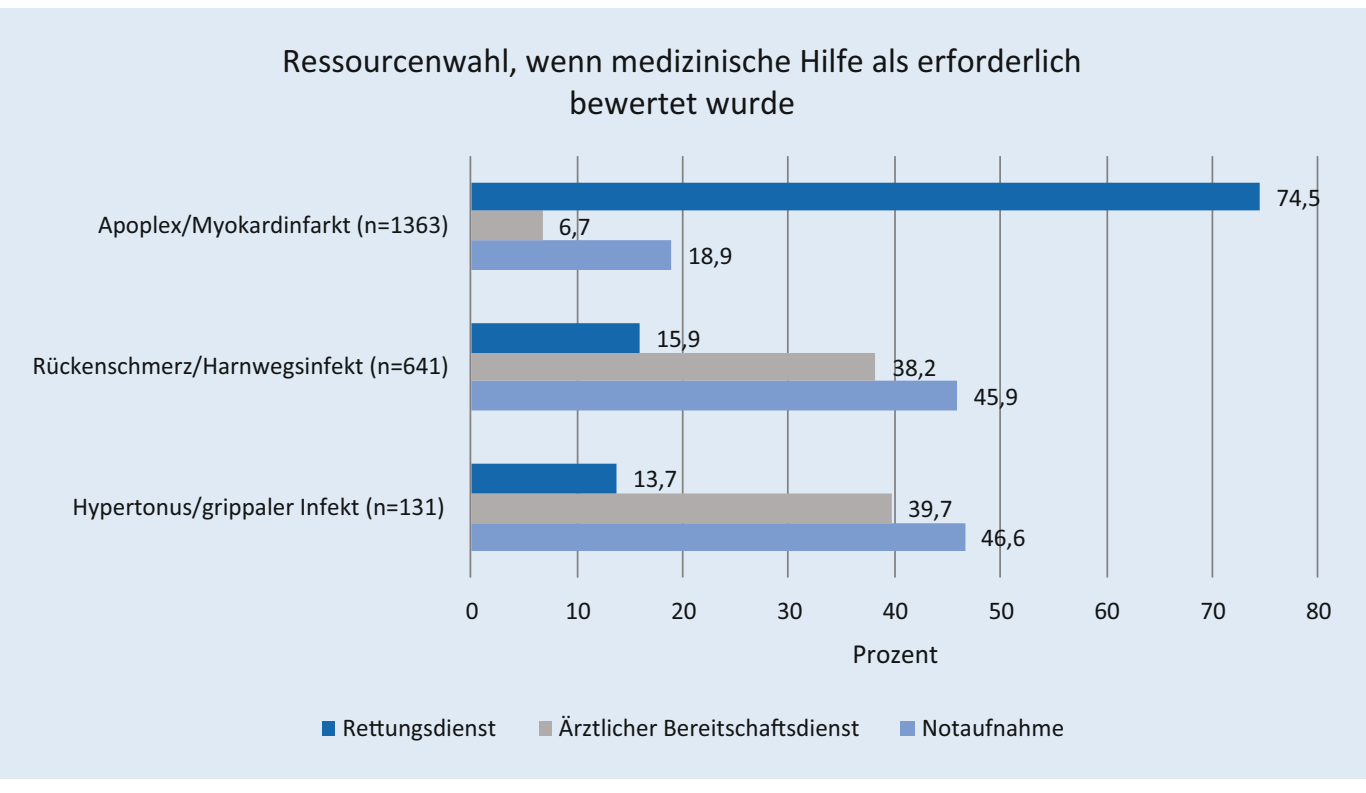

Abb. $3 \triangleleft$ Ressourcenwahl, wenn medizinische Hilfe als erforderlich bewertet wurde; dargestellt ist die durch die Befragten getroffene Auswahl der Ressource aggregiert nach Dringlichkeit der Fälle in die 3 Kategorien "sehr dringlich" (Apoplex und Myokardinfarkt), „mittel dringlich“ (Rückenschmerz und Harnwegsinfekt) und "nicht/leicht dringlich" (Hypertonus und grippaler Infekt)

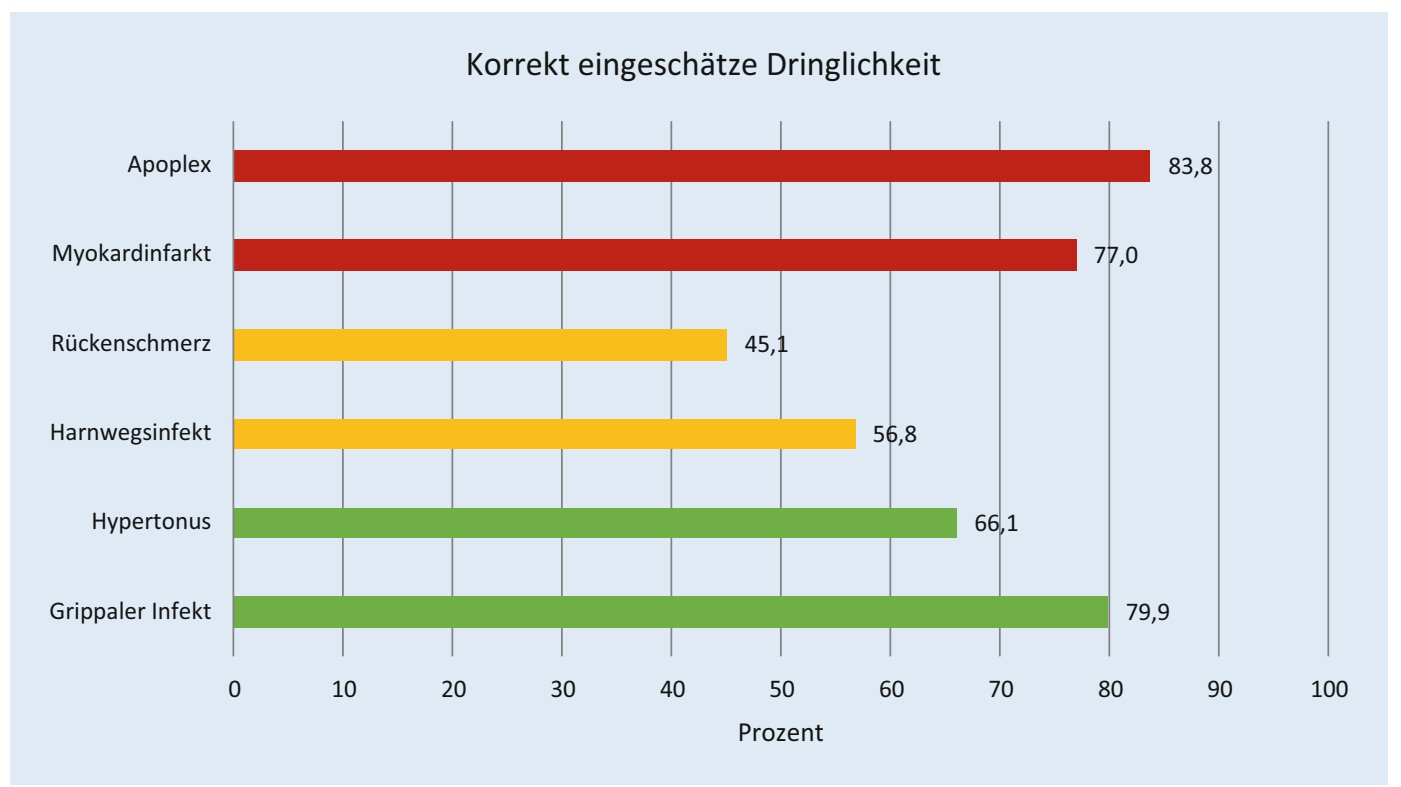

Abb. $4 \varangle$ Subjektive Dringlichkeitseinschätzung in Relation zur tatsächlichen Dringlichkeit. Anteil der durch die Befragten korrekt eingeschätzten Dringlichkeit der verschiedenen Szenarien dass sie den ÄBD (39,7\%) kontaktieren. Die verminderte Nutzung einer Ressource führt konsekutiv zu einer Belastung der anderen Ressourcen [10, 14, 27, 30]. Sowohl im Rettungsdienst als auch in der Notaufnahme war in den letzten Jahren ein deutlich gesteigertes Patientenaufkommen zu verzeichnen [1, 19, 26, 27, 29]. Diese Mehrbelastungen sind mit erheblichen Kosten verbunden und bergen das Risiko, dass die Kapazitäten für die Behandlungen von lebensbedrohlich erkrankten Personen nicht ausreichen [15, 26].

Neben der Fehleinschätzung der Dringlichkeit könnte die geringe Nut- zung der Ressource „ärztlicher Bereitschaftsdienst" auch darin liegen, dass jeder Vierte auch auf Nachfrage diese Ressource nicht kannte. Und nur $18 \%$ der Befragten konnten eine korrekte Telefonnummer zur Alarmierung des ÄBD nennen. Dies ist eine Steigerung der Bekanntheit im Vergleich zu einer 2013 (ein Jahr nach der Einführung der 116117 [7]) in der Westpfalz durchgeführten Telefonbefragung, bei der nur 2\% die Nummer 116117 kannten [16]. Doch auch 5 Jahre später und nach großen Werbekampagnen ist die Nummer weitgehend unbekannt.
Der Bevölkerung ist mehrheitlich nicht bewusst, welches Einsatzspektrum der Rettungsdienst bzw. der ärztliche Bereitschaftsdienst übernimmt $[16,27]$. Im Sinne der Steigerung der Gesundheitskompetenz sollten Maßnahmen ergriffen werden, um das Wissen zu Gesundheit und Krankheit und Handlungskompetenzen in der Bevölkerung zu stärken, damit diese befähigt ist, eigenständig adäquate Entscheidungen zu treffen [3, 28]. Die Schulungsmaßnahmen müssen gleichzeitig die Über- und Unterschätzung adressieren und es besteht das Risiko, dass die Fehleinschätzung nur 


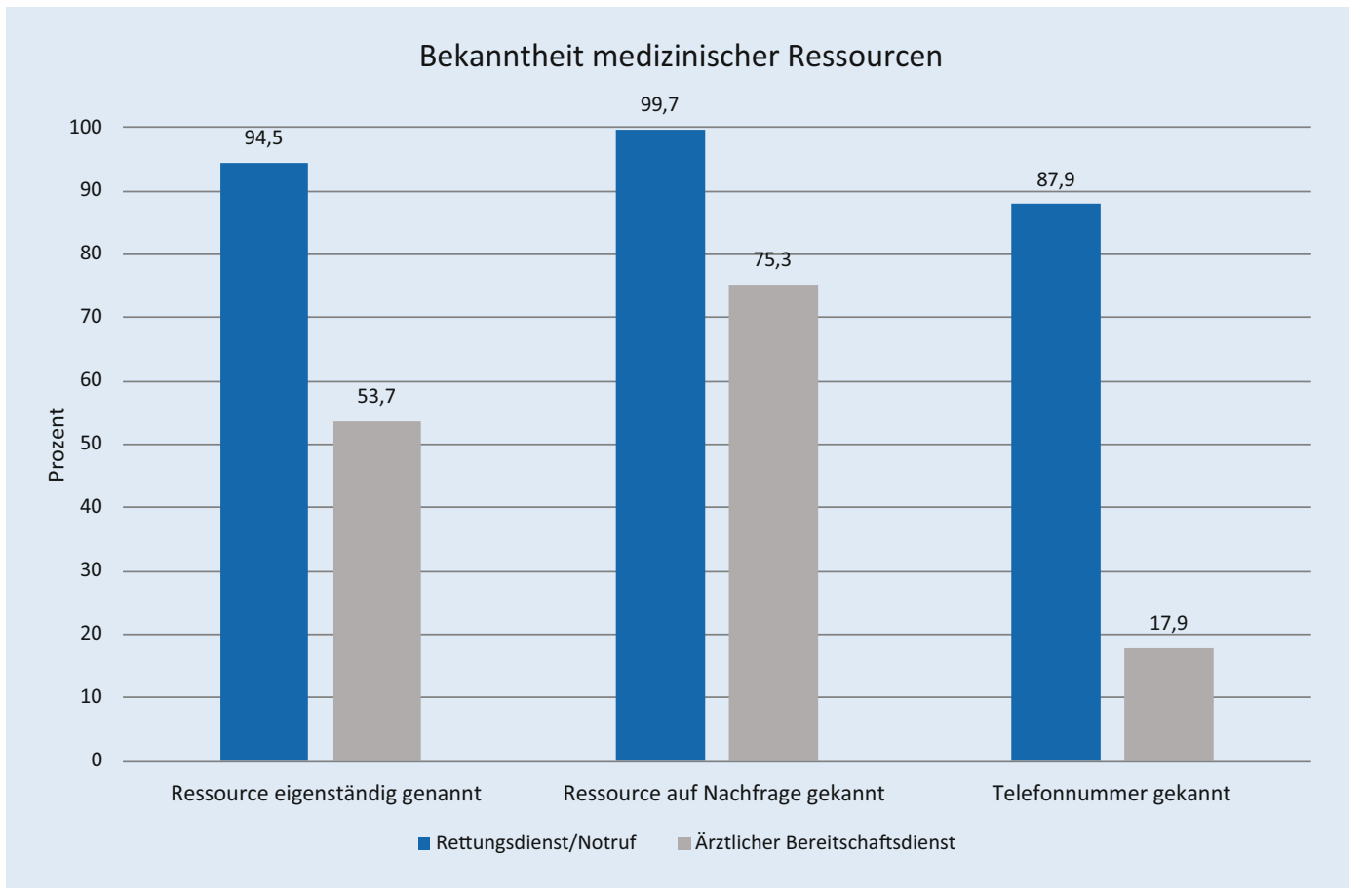

Abb. $5 \triangleleft$ Bekanntheit medizinischer Ressourcen in eine Richtung verschoben und hier verstärkt wird.

Wichtig scheint, dass neben einem größeren Wissen über die verschiedenen Ressourcen auch ein höheres Bewusstsein für unterschiedliche Dringlichkeiten geschaffen wird $[16,19]$. Hierbei muss davon ausgegangen werden, dass der Schulungseffekt erst mit deutlicher Verzögerung eintritt $[1,14,16]$ und enorme Anstrengungen erforderlich sind, um die gewünschten Effekte zu erzielen.

Neben der Steigerung der Gesundheitskompetenz ist darüber hinaus eine stärkere Synergie der akutmedizinischen Ressourcen zu fordern. Die eigenständige Zuordnung durch Patienten zu einem der 3 akutmedizinischen Versorgungssysteme wurde als suboptimal identifiziert $[1,4,10,14,19,20,27,30]$. Daher hat das Bundesministerium für Gesundheit einen Referentenentwurf vorgelegt, der eine Neugliederung der Notfallversorgung in Deutschland vorsieht [2]. Dieser beinhaltet eine engere Verknüpfung der unterschiedlichen Partner in der Patientenversorgung; diese sektorenübergreifende Zusammenarbeit wird stark diskutiert [10, 27]. Zentrales Element des Maßnahmenbündels ist ein gemeinsames Notfallleitsystem, das von der Bevölkerung bei medizinischen Akutfäl- len kontaktiert werden kann und die richtige Ressource zuordnet [2].

Die Etablierung einer gemeinsamen Telefonnummer für Rettungsdienst und ÄBD scheint ein Lösungsweg zu sein [1, $10,16,18,21,27]$. Dies wurde in einigen Regionen Deutschlands als Modellprojekt realisiert und ist in vielen Ländern Europas seit Jahren erfolgreich etabliert $[10,14,17,22]$. Über eine solche Nummer kann nach einer standardisierten Abfrage eine Zuweisung zum geeigneten System erfolgen $[1,10]$. Somit könnte man auch Patienten, die die Dringlichkeit der Situation fehleinschätzen, schnell der richtigen Versorgungsform zukommen lassen $[1,10,21]$. Dies würde die Patienten von der Verantwortung entlasten, in einer für sie belastenden Situation diese schwierige Entscheidung treffen zu müssen [27]. Zusätzlich wird eine erheblich geringere Fehlnutzung der einzelnen Ressourcen erwartet. Neben einer gemeinsamen Telefonnummer besteht auch die Möglichkeit, ein gemeinsames Abfragetool mit intelligenter Verknüpfung zu entwickeln, das unabhängig von der gewählten Nummer eine schnelle Zuweisung sicher ermöglicht [27]. Dabei ist es essenziell, dass ein einheitliches Abfragesystem zuvorderst darauf abzielt, zeitkritische Notfälle unmittelbar $\mathrm{zu}$ identifizieren, um in diesen Fällen schnellstmöglich den Rettungsdienst zu alarmieren. Derzeit werden mehrere Abfragetools international evaluiert $[6,10,14,18,23]$.

\section{Limitationen der Studie}

Einschränkend muss beachtet werden, dass das Design einer Telefonumfrage immer nur eine Stichprobe der Bevölkerung darstellt. Durch das Gabler-HäderDesign wurde versucht, die Übertragbarkeit der Studienergebnisse zu erhöhen. Es lässt sich vermuten, dass die Entscheidung der Dringlichkeit und die Ressourcenwahl von verschiedenen Faktoren beeinflusst werden (z. B. sozioökonomischer und kultureller Hintergrund, Vorerkrankungen, soziales Netzwerk und emotionale Faktoren). Es kann nicht ausgeschlossen werden, dass sich die Befragten in einer tatsächlichen Akutsituation anders entscheiden, als sie es nach der telefonischen Schilderung des Szenarios getan haben.

\section{Fazit}

- $88 \%$ der Befragten kennen die Nummer des Rettungsdiensts, aber nur $18 \%$ kennen die Nummer des ärztlichen Bereitschaftsdiensts. 
- Die Dringlichkeit einer geschilderten Apoplex- und Myokardinfarktsymptomatik wird von jedem Vierten unterschätzt und 2-5\% der Stichprobe halten hier kurzfristige medizinische Hilfe für nicht erforderlich.

- Bei Erkrankungen mittlerer Dringlichkeit würden mehr Befragte eigenständig in die Notaufnahme gehen, als dass sie den ärztlichen Bereitschaftsdienst alarmieren würden.

- Der Kenntnisstand über die verschiedenen Ressourcen bei medizinischen Akutfällen und die Fähigkeit, die Dringlichkeit dieser Situationen adäquat einzuschätzen, scheint in der deutschen Bevölkerung nicht ausreichend zu sein. Eine Steigerung der Gesundheitskompetenz sollte angestrebt werden.

- Eine geeignete Lösung zur adäquaten Zuordnung der Ressourcen Rettungsdienst und ärztlicher Bereitschaftsdienst scheint eine gemeinsame Koordination mit einheitlichem Abfragetool zu sein.

\section{Korrespondenzadresse}

\section{Dr. med. Bibiana Metelmann}

Klinik für Anästhesiologie, Anästhesie, Intensiv-, Notfall- und Schmerzmedizin, Universitätsmedizin Greifswald Ferdinand-Sauerbruch-Straße, 17475 Greifswald, Deutschland bibiana.metelmann@uni-greifswald.de

Danksagung. Stellvertretend für die Mitarbeiter der GESIS, Leibniz-Institut für Sozialwissenschaften, gilt unser großer Dank Frau Dr. Lydia Repke, Herrn Dr. Matthias Sand und Herrn Prof. Dr. Michael Braun für ihre umfangreiche Beratung und die Bereitstellung der Telefonnummern. Wir bedanken uns bei Herrn Dr. Paul Wolf von der Hochschule Stralsund. Den Mitgliedern der Forschungs-AG Notfallmedizin der UMG danken wir für die konstruktiven Ideen.

Funding. Open Access funding enabled and organized by Projekt DEAL.

\section{Einhaltung ethischer Richtlinien}

Interessenkonflikt. B. Metelmann, P. Brinkrolf, M. Kliche, M. Vollmer, K. Hahnenkamp und C. Metelmann geben an, dass kein Interessenkonflikt besteht.

Diese retrospektive Studie erfolgte nach Konsultation der zuständigen Ethikkommission und im Einklang mit nationalem Recht.
Open Access. Dieser Artikel wird unter der Creative Commons Namensnennung 4.0 International Lizenz veröffentlicht, welche die Nutzung, Vervielfältigung, Bearbeitung, Verbreitung und Wiedergabe in jeglichem Medium und Format erlaubt, sofern Sie den/die ursprünglichen Autor(en) und die Quelle ordnungsgemäß nennen, einen Link zur Creative Commons Lizenz beifügen und angeben, ob Änderungen vorgenommen wurden.

Die in diesem Artikel enthaltenen Bilder und sonstiges Drittmaterial unterliegen ebenfalls der genannten Creative Commons Lizenz, sofern sich aus der Abbildungslegende nichts anderes ergibt. Sofern das betreffende Material nicht unter der genannten Creative Commons Lizenz steht und die betreffende Handlung nicht nach gesetzlichen Vorschriften erlaubt ist, ist für die oben aufgeführten Weiterverwendungen des Materials die Einwilligung des jeweiligen Rechteinhabers einzuholen.

Weitere Details zur Lizenz entnehmen Sie bitte der Lizenzinformation auf http://creativecommons.org/ licenses/by/4.0/deed.de.

\section{Literatur}

1. Enneker-Forum-Falkenstein, ThinkTank der ClausEnneker-Stiftung (2019) Antworten auf die aktuellen Fragen zur zukünftigen notfallmedizinischen Versorgung der Bevölkerung in Deutschland. Notfall Rettungsmed 22:733-737. https://doi.org/ 10.1007/s10049-019-00650-1

2. Bundesministerium fürGesundheit(2020) Entwurf eines Gesetzes zur Reform der Notfallversorgung

3. Abel T, Sommerhalder K (2015) Gesundheitskompetenz/Health Literacy: Das Konzept und seine Operationalisierung. Bundesgesundheitsblatt Gesundheitsforschung Gesundheitsschutz 58:923-929. https://doi.org/10.1007/s00103015-2198-2

4. Beivers A (2020) Neujustierung der Notfallversorgung durch sektorenübergreifende Notfallzentren. In: Pfannstiel MA, Jaeckel R, Da-Cruz P (Hrsg) Market Access im Gesundheitswesen: Hürden und Zugangswege zur Gesundheitsversorgung. Springer, Wiesbaden, S253-267

5. Booker MJ, Purdy S, Shaw ARG (2017) Seeking ambulance treatment for "primary care" problems. Aqualitative systematic review of patient, carerand professional perspectives. BMJ Open 7:e16832. https://doi.org/10.1136/bmjopen-2017-016832

6. Brasseur E, Servotte J-C, Donneau A-F et al (2019) Triage for out-of-hours primary care calls: a reliability study of a new French-language algorithm, the SALOMON rule. Scand J Prim Health Care 37:227-232. https://doi.org/10.1080/ 02813432.2019 .1608057

7. Breckner A, Roth C, Wensing $M$ et al (2020) Quo vadis 116117 ? Bundesweiter Überblick über den Status quo und aktuelle Veränderungen. Gesundheitswesen 82:324-327. https://doi.org/ 10.1055/a-1075-2330

8. Brokmann JC, Pin M, Bernhard M et al (2019) Neustrukturierung der stationären Notfallversorgung: Was ändert sich? Anaesthesist 68:261-269. https://doi.org/10.1007/s00101-019-0588-9

9. Geissler A, Quentin W, Busse R (2017) Umgestaltung der Notfallversorgung. Internationale Erfahrungen und Potenziale für Deutschland. In: Klauber J, Geraedts M, Friedrich J, Wasem J (Hrsg)
Krankenhaus-Report 2017. Schwerpunkt: Zukunft gestalten. Schattauer, Stuttgart, S41-59

10. Graf von Stillfried D, Czihal T, Meer A (2019) Sachstandsbericht: Strukturierte medizinische Ersteinschätzung in Deutschland (SmED). Notfall Rettungsmed 22:578-588. https://doi.org/10. 1007/s10049-019-0627-8

11. Häder S, Häder M, Schmich P (2019) Telefonumfragen in Deutschland. Springer, Wiesbaden

12. International Chamber of Commerce (2016) ICC/ESOMAR international code on market, opinion and social research and data analytics. https://www.esomar.org/uploads/ public/knowledge-and-standards/codes-andguidelines/ICCESOMAR_Code_English_.pdf. Zugegriffen:28. Apr. 2018

13. Kirkby HM, Roberts LM (2012) Inappropriate 999 calls. An online pilot survey. Emerg Med J 29:141-146. https://doi.org/10.1136/emj.2010. 092346

14. Kumle B, Hirschfeld-Warneken A, Darnhofer l et al (2019) Telefon-Triage und klinische Ersteinschätzung in der Notfallmedizin zur Patientensteuerung. Notfall Rettungsmed 22:568-577. https:// doi.org/10.1007/s10049-019-0622-0

15. Lechleuthner A, Wesolowski M, Brandt S (2019) Gestuftes Versorgungssystem im Kölner Rettungsdienst. Notfall Rettungsmed 22:598-607. https:// doi.org/10.1007/s10049-019-00644-z

16. Luiz T, Dittrich S, Pollach G et al (2017) Kenntnisstand der Bevölkerung über Leitsymptome kardiovaskulärer Notfälle und Zuständigkeit und Erreichbarkeit von Notrufeinrichtungen. Ergebnisse der KZEN-Studie in der Westpfalz. Anaesthesist 66:840-849. https://doi.org/10.1007/s00101017-0367-4

17. Meer A, Simonin C, Trapp A et al (2003) Einfluss der medizinischen computerassistierten Telefontriage auf das Patientenverhalten: erste Erfahrungen in der Schweiz. Schweiz Arzteztg 84(41):2160-2165

18. Meer A, Gwerder T, Duembgen L et al (2012) Is computer-assisted telephone triage safe? A prospective surveillance study in walk-in patients with non-life-threatening medical conditions. Emerg Med J 29:124-128. https://doi.org/10. 1136/emj.2009.080614

19. Rau R, Mensing M, Brand H (2006) „Medizinische Notfalldienste aus Nutzersicht": Ein Beitrag zur Versorgungsforschung durch CATI-Befragung der Bevölkerung. Med Klin 101:37-47.https://doi.org/ 10.1007/s00063-006-1006-z

20. Reinhold AK, Greiner F, Schirrmeister W et al (2020) DerNotfall "geht" ins Krankenhaus: Eine Befragung von Patienten mit niedriger Dringlichkeit in einer Notfallaufnahme mit regionaler Alleinstellung. Med Klin Intensivmed Notfmed. https://doi.org/ 10.1007/s00063-020-00681-4

21. Roivainen $P$, Hoikka $M J$, Raatiniemi $L$ et al (2020) Telephone triage performed by nurses reduces non-urgent ambulance missions: a prospective observational pilot study in Finland. Acta Anaesthesiol Scand. https://doi.org/10.1111/ aas. 13542

22. Roßbach-Wilk E, Beivers A, Dodt C (2019) Patientensteuerung von Notfallpatienten mit niedrigem Gesundheitsrisiko. Notfall Rettungsmed 22:561-567. https://doi.org/10.1007/s10049019-0618-9

23. Roth C, Breckner A, Paulus J et al (2020) Implementation of a standardized initial assessment for demand management in outpatient emergency care in Germany: early qualitative process evaluation. JMIR Form Res 4:e18456. https://doi.org/10. 2196/18456 
24. Ruud SE, Hjortdahl P, Natvig B (2016) Is it a matter of urgency? A survey of assessments by walk-in patients and doctors of the urgency level of their encounters at a general emergency outpatient clinic in Oslo, Norway. BMC Emerg Med 16:22. https://doi.org/10.1186/s12873-016-0086-1

25. Scherer M, Lühmann D, KazekA etal (2017) Patients attending emergency departments. Dtsch Arztebl Int 114:645-652. https://doi.org/10.3238/arztebl. 2017.0645

26. Schmiedhofer $M$, Möckel $M$, Slagman A et al (2016) Patient motives behind low-acuity visits to the emergency department in Germany. A qualitative study comparing urban and rural sites. BMJ Open 6:e13323. https://doi.org/10.1136/ bmjopen-2016-013323

27. Sefrin $P$ (2018) Neuordnung der Notfallversorgung im ambulanten/präklinischen Bereich. Notarzt 34:132-139. https://doi.org/10.1055/a-06042527

28. Seidel G, Meyer A, Lander J et al (2020) Facetten von Gesundheitskompetenz. Präv Gesundheitsf 15:65-72. https://doi.org/10.1007/s11553-01900736-1

29. Sieber F, Kotulla R, Urban B et al (2020) Entwicklung der Frequenz und des Spektrums von Rettungsdiensteinsätzen in Deutschland. Notfall
Rettungsmed 23:490-496. https://doi.org/10. 1007/s10049-020-00752-1

30. Somasundaram R, Geissler A, Leidel BA et al (2016) Beweggründe für die Inanspruchnahme von Notaufnahmen - Ergebnisse einer Patientenbefragung. Gesundheitswesen. https://doi.org/ 10.1055/s-0042-112459

31. Toloo G-S, Aitken P, Crilly J et al (2016) Agreement between triage category and patient's perception of priority in emergency departments. Scand J Trauma ResuscEmerg Med 24:126. https://doi.org/ 10.1186/s13049-016-0316-2

\section{Lesen Sie die Medizinische Klinik - Intensivmedizin und Notfallmedizin online auf SpringerMedizin.de}

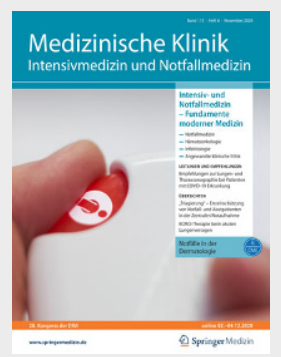

Auf SpringerMedizin.de erhalten Sie Zugang zu allen elektronisch verfügbaren Ausgaben und dem CME-Angebot Ihrer Zeitschrift - unabhängig davon, seit wann Sie die Medizinische Klinik - Intensivmedizin und Notfallmedizin abonniert haben. Außerdem können Sie die Zeitschrift als ePaper bequem auf Ihrem Tablet lesen.

Alle Inhalte der Zeitschrift finden Sie unter

www.springermedizin.de/mk-im

\section{So einfach erhalten Sie Zugang zum Online Archiv}

Registrieren Sie sich einmalig auf www.springermedizin.de/register

Geben Sie dabei Ihre Einheitliche Fortbildungsnummer (EFN) an.

- Ihr Benutzername entspricht Ihrer E-Mail-Adresse, Ihr Passwort können Sie frei wählen und später jederzeit unter „Mein Profil“ ändern.

- Falls Sie bereits ein (Print-) Abonnement bei uns haben, geben Sie bei der Registrierung die Lieferadresse Ihrer Zeitschrift an. Damit wird Ihr Abo-Zugang auf springermedizin.de freigeschaltet.
Sind Sie bereits bei SpringerMedizin.de registriert? Dann wird Ihr Zeitschriftenabonnement automatisch Ihrem Online-Nutzerkonto hinzugefügt. Sollten die Angaben Ihres OnlineAccounts nicht eindeutig mit den Angaben Ihres ZeitschriftenAbonnements übereinstimmen, kann die Zuordnung nicht sicher erfolgen. In diesem Fall und bei allen anderen Fragen zum Online-Zugang kontaktieren Sie bitte unseren Kundenservice unter: Kundenservice@springermedizin.de

Telefonisch erreichen Sie den Kundenservice montags bis freitags von 9.00 bis 17.00 Uhr kostenfrei unter $\mathbf{0 8 0 0 - 7 7 ~} 80777$ sowie gebührenpflichtig aus dem Ausland unter +49 30884293600 